\title{
Measurement of acoustic nonlinearity parameter using focused ultrasound detected by a concave receiver
}

\author{
Shigemi Saito \\ Faculty of Marine Science and Technology, Tokai University, \\ 3-20-1, Orido, Shimizu, 424 Japan
}

(Received 28 September 1994)

\begin{abstract}
The measurement method for the acoustic nonlinearity parameter $B / A$ of a liquid sample inserted in the focal region has been presented, where the acoustic wave of focused Gaussian beam observed with a concave receiver which is set perpendicular to the acoustic axis within the postfocal region is utilized. Both the amplitudes and phase difference of the fundamental and second harmonic components in the signal detected with a concave receiver set at the position, where the obtained signal amplitude attains the maximum for each the case with and without insertion of the sample, are transformed to the values expected for the axial pressure at the range corresponding to the maximum position for the case with the insertion of the sample. Then the previously presented method utilizing the axial pressure turns out to be applicable. Using a $1.9-\mathrm{MHz}$ focusing source and a concave receiver, the confirmation of validity of the present method was intended through the experiment employing the samples of alcohol and glycerin. The result verified the capability of the present method to measure the $B / A$.
\end{abstract}

Keywords: Nonlinear acoustics, Focused ultrasound, Second harmonic, Acoustic microscope, Nonlinearity parameter, Finite amplitude method

PACS number: 43. 25. Cb, 43. 25. Zx, 43. 25. Jh, 43. 80. Ev, 43. 35. Sx

\section{INTRODUCTION}

Acoustic nonlinearity parameter $B / A$ describes the magnitude of the quadratic dependence of the acoustic pressure on the density of medium. The equation of sound propagation includes the $B / A$ in the nonlinear term. This parameter is very important to estimate nonlinear distortion and attenuation of the sound with moderate or large amplitude. Further it has been suggested that this parameter is available for material characterization of the medium. So the measurement method is also of great interest.

Utilizing the property of the second harmonic component generated by the nonlinear propagation of focused sound, the present author proposed and experimented a method to measure the acoustic nonlinearity parameter $B / A$ of a liquidlike medium inserted in the focal region by observing the axial acoustic pressure beyond the sample with a point receiver. ${ }^{1)}$ Small volume samples, for example, of $0.5 \mathrm{ml}$ were measured using a $1.9-\mathrm{MHz}$ focusing source. $^{2)}$ In this method, the measurement of a parameter which has been chosen as a parameter relatively insensitive to the sample thickness and attenuation is experimentally evaluated. Then the value of $B / A$ is determined through the comparison of the experimental parameter with the theoretically calculated one. In this procedure, the phase parameter which defines the phase delay of the second harmonic pressure relative to the fundamental pressure has been utilized to selectively detect the second harmonic component generated within the focal region..$^{3,4)}$

On the other hand, acoustic microscopes have been applied to various kinds of acoustic characterizations and measurements, where high-frequency focused sounds are employed to obtain high spatial 
resolution. Although the nonlinear phenomena must take place in the acoustic microscope, the phenomena are never used for acoustical measurements. To apply acoustic microscopes to the $B / A$ measurement which is mentioned above, the properties of the fundamental and second harmonic components of the focused Gaussian beam detected by a concave receiver have been analyzed theoretically and experimentally in the previous paper.5) The result shows that, when the receiver is set so that the fundamental amplitude of the received signal attains the maximum, the second harmonic amplitude also takes the maximum and further the amplitudes and phase difference of two components can be simply related to those of the axial pressure. The phase difference is called phase parameter. Based on this analysis, utilizing the fundamental and second harmonic components detected by a concave receiver, a method to measure the nonlinearity parameter $B / A$ of liquidlike samples inserted into the focal region is proposed in this paper. A Gaussian beam is assumed for the focused sound, where the successive approximation solution for the governing equation is adopted for the analytical model. The experiment to validate the theory and to confirm the possibility of measuring the $B / A$ is conducted at relatively low frequency of $1.9 \mathrm{MHz}$, for convenience.

\section{MEASUREMENT METHOD FOR $B / A$}

2.1 Transform to Axial Parameters

Suppose that a spherical focusing source of focal length $D$ set at $z=0$ emits a sinusoidal sound of angular frequency $2 \pi f=\omega$ along the $z$ axis as shown in Fig. 1, where the medium is water. The sound source is assumed to be a Gaussian source with on-

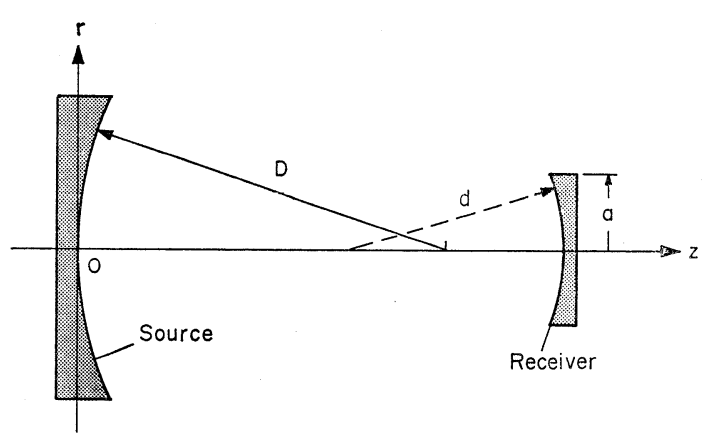

Fig. 1 Geometry and notation. source pressure amplitude of $p_{0} \exp \left(-\xi r^{2}\right)$. The focused sound is detected by a concave receiver whose curvature and aperture radii are $d$ and $a$, respectively. Integrating the acoustic pressure on the receiving surface, the receiver output voltage in the case of free field and that in the case with the insertion of the sample are expressed as $P_{\mathrm{W}}=P_{1 \mathrm{w}}(z)$ $\times \exp (-j \omega \tau)+P_{2 \mathrm{~W}}(z) \exp (-j 2 \omega \tau)$ and $\boldsymbol{P}_{\mathrm{S}}=\boldsymbol{P}_{1 \mathrm{~S}}(z)$ $\times \exp (-j \omega \tau)+P_{2 \mathrm{~S}}(z) \exp (-j 2 \omega \tau)$, respectively, as noted in the previous paper. ${ }^{5)}$ Here $\tau$ is the retarded time $t-z / c_{\mathrm{w}}$, where $t$ is the time, and $c_{\mathrm{w}}$ is the sound speed in water. The range to make both $\boldsymbol{P}_{1 \mathrm{~W}}$ and $\boldsymbol{P}_{2 \mathrm{~W}}$ maximum is named $z_{\mathrm{w}}$, and the range for the maximum $P_{1 \mathrm{~S}}$ and $P_{2 \mathrm{~S}}$ is named $z_{\mathrm{s}}$.

The theoretical values for the fundamental and second harmonic components of the axial acoustic pressure are easily obtained. In Ref. 1), a method to measure $B / A$ through observing both the amplitudes of the fundamental and second harmonic components and the phase parameter for the axial pressure was presented. The validity of this method was confirmed through the experiments. Consequently, if the voltage of the signal detected by the concave receiver can be transformed to the amplitude and phase of the axial pressure, the method of Ref. 1) can be directly applied. Since both the waveform distortion and phase parameter at the range for the maximum amplitude are in simple relations with those of the acoustical pressure, the measured data of the concave receiver output may be easily transformed to the axial parameters.

When the sample is not immersed in the focused field, the fundamental and second harmonic components of the concave receiver output at the range, where the maximum amplitude is observed, can be obtained by substituting $z=z_{\mathrm{w}}$ into Eqs. (5) and (6) of Ref. 5):

$$
\begin{gathered}
P_{1 \mathrm{w}}\left(z_{\mathrm{w}}\right)=-\frac{k_{\mathrm{w}} p_{0}}{2 g\left(z_{\mathrm{w}}\right) h\left(z_{\mathrm{w}}\right)} \\
\cdot \exp \left(-\alpha_{\mathrm{w}} z\right)\left[\exp \left\{j g\left(z_{\mathrm{w}}\right) a^{2}\right\}-1\right], \\
P_{2 \mathrm{w}}\left(z_{\mathrm{w}}\right)= \\
\frac{\beta_{\mathrm{w}} k_{\mathrm{w}}{ }^{3} p_{0}{ }^{2} \exp \left(-4 \alpha_{\mathrm{w}} z_{\mathrm{w}}\right)}{8 \rho_{\mathrm{w}} c_{\mathrm{w}}{ }^{2} g\left(z_{\mathrm{w}}\right) h\left(z_{\mathrm{w}}\right)}\left[\exp \left\{j 2 g\left(z_{\mathrm{w}}\right) a^{2}\right\}-1\right] \\
\cdot \int_{0}^{z_{\mathrm{w}}} \frac{\exp \left(2 \alpha_{\mathrm{w}} z^{\prime}\right)}{h\left(z^{\prime}\right)} d z^{\prime},
\end{gathered}
$$

where $k_{\mathrm{w}}$ is the wavenumber of the fundamental in water $\omega / c_{\mathrm{w}}, \alpha_{\mathrm{w}}$ is the attenuation coefficient in water at the fundamental frequency, $\rho_{\mathrm{w}}$ is the density of water, and $\beta_{\mathrm{w}}$ is the acoustic nonlinearity parameter 


\section{S. SAITO: $B / A$ MEASUREMENT USING FOCUSED ULTRASOUND}

$1+(B / A)_{\mathrm{w}} / 2$ of water. In addition, $h(z)=2 \xi_{z}-$ $j k_{\mathrm{w}}(1-z / D)$, and $g(z)=k_{\mathrm{w}}\left[1+j k_{\mathrm{w}} / h(z)\right] / 2 z-k_{\mathrm{w}} / 2 d$.

The output for the case with the insertion of the sample of thickness $L$ is obtained when putting $z=z_{\mathrm{s}}\left[=z_{\mathrm{w}}-\left(c / c_{\mathrm{w}}-1\right) L\right]$ in Eqs. (13) and (14) of Ref. 5 ), where $c$ is the sound speed in the sample.

$$
\begin{aligned}
P_{1 \mathrm{~S}}\left(z_{\mathrm{s}}\right)= & -\frac{k_{\mathrm{w}} T_{\mathrm{I} 1} T_{\mathrm{O} 1}}{2 g\left(z_{\mathrm{w}}\right) h\left(z_{\mathrm{w}}\right)} \\
& \cdot p_{0} \exp \left[-\alpha_{\mathrm{w}}\left(z_{s}-L\right)-\alpha_{1} L\right] \\
& \cdot\left[\exp \left\{j g\left(z_{\mathrm{w}}\right) a^{2}\right\}-1\right] \\
P_{2 \mathrm{~S}}\left(z_{\mathrm{s}}\right)= & \frac{k_{\mathrm{w}}^{3} p_{0}^{2}}{8 \rho_{\mathrm{w}} c_{\mathrm{w}}^{2} g\left(z_{\mathrm{w}}\right) h\left(z_{\mathrm{w}}\right)} \\
& \cdot \exp \left[-4 \alpha_{\mathrm{w}}\left(z_{\mathrm{s}}-L\right)-\alpha_{2} L\right] \\
& \cdot\left[\exp \left\{j 2 g\left(z_{\mathrm{w}}\right) a^{2}\right\}-1\right] \\
& \cdot\left\{\beta_{\mathrm{w}} T_{\mathrm{I} 2} T_{\mathrm{O} 2} \int_{0}^{Z_{\mathrm{I}}} \frac{\exp \left(2 \alpha_{\mathrm{w}} z^{\prime}\right)}{h\left(z^{\prime}\right)} d z^{\prime}\right. \\
+ & \beta T_{\mathrm{I} 1}^{2} T_{\mathrm{O} 2} \frac{\rho_{\mathrm{w}} c_{\mathrm{w}}{ }^{3}}{\rho c^{3}} \exp \left(2 \alpha_{\mathrm{w}} z_{\mathrm{I}}\right) \\
& \cdot \int_{0}^{L} \frac{\exp \left[\left(\alpha_{2}-2 \alpha_{1}\right) z^{\prime}\right]}{h\left(z_{\mathrm{I}}+c z^{\prime} / c_{\mathrm{w}}\right)} d z^{\prime}+\beta_{\mathrm{w}} T_{\mathrm{I1}}{ }^{2} T_{01}{ }^{2} \\
& \cdot \exp \left[2 \alpha_{\mathrm{w}} z_{\mathrm{I}}+\left(\alpha_{2}-2 \alpha_{1}\right) L\right] \\
& \left.\cdot \int_{0}^{z_{\mathrm{s}}-z_{\mathrm{o}}} \frac{\exp \left(2 \alpha_{\mathrm{w}} z^{\prime}\right)}{h\left(z^{\prime}+z_{\mathrm{I}}+c L / c_{\mathrm{w}}\right)} d z^{\prime}\right\}
\end{aligned}
$$

where $\rho$ and $\beta=1+(B / A) / 2$ are the density and nonlinearity parameter of sound in the sample layer, respectively. The attenuation coefficient in the sample is assumed to be $\alpha_{1}$ at the fundamental frequency and $\alpha_{2}$ at the second harmonic frequency. The transmission coefficients of acoustic pressure $T_{\text {In }}$ and $T_{\mathrm{O} n}(n=1,2)$ are determined as Eqs. (15) and (16) of Ref. 5). Further, $z_{\mathrm{I}}=D-L / 2$ and $z_{0}=$ $D+L / 2$. Note that $z_{\mathrm{a}}$ in Eqs. (13) and (14) of Ref. 5) has been replaced with $z_{\mathrm{w}}$, because $z_{\mathrm{a}}=z_{\mathrm{w}}$ when $z=z_{\mathrm{s}}$.

The axial pressures are rewritten as $p_{1 \mathrm{w}}(0, z)=$ $p_{1 \mathrm{w}}(z), \quad p_{2 \mathrm{w}}(0, z)=p_{2 \mathrm{w}}(z), \quad p_{1 \mathrm{~s}}(0, z)=p_{1 \mathrm{~s}}(z), \quad$ and $p_{2 \mathrm{~s}}(0, z)=p_{2 \mathrm{~s}}(z)$ for simplicity. Eliminating $p_{0} / h\left(z_{\mathrm{w}}\right)$ by use of Eq. (1) and the expression for $p_{1 \mathrm{w}}\left(z_{\mathrm{w}}\right)$ derived from Eq. (3) of Ref. 5),

$$
\boldsymbol{P}_{1 \mathrm{w}}\left(z_{\mathrm{w}}\right)=\frac{\exp \left[j g\left(z_{\mathrm{w}}\right) a^{2}\right]-1}{j 2 g\left(z_{\mathrm{w}}\right)} p_{1 \mathrm{w}}\left(z_{\mathrm{w}}\right) .
$$

Comparison of the substitutions of $z=z_{\mathrm{w}}$ and $z=z_{\mathrm{s}}$ into Eq. (3) of Ref. 5) leads to

$$
p_{1 \mathrm{w}}\left(z_{\mathrm{w}}\right)=\frac{\exp \left[-\alpha_{\mathrm{w}}\left(z_{\mathrm{w}}-z_{\mathrm{s}}\right)\right]}{\zeta} p_{1 \mathrm{w}}\left(z_{\mathrm{s}}\right),
$$

where

$$
\zeta=\frac{h\left(z_{\mathrm{w}}\right)}{h\left(z_{\mathrm{s}}\right)}
$$

Substituting Eq. (6) into Eq. (5), one derives

$$
\begin{aligned}
P_{1 \mathrm{w}}\left(z_{\mathrm{w}}\right)= & \frac{\exp \left[j g\left(z_{\mathrm{w}}\right) a^{2}\right]-1}{j 2 \zeta g\left(z_{\mathrm{w}}\right)} \\
& \cdot \exp \left[-\alpha_{\mathrm{w}}\left(z_{\mathrm{w}}-z_{\mathrm{s}}\right)\right] p_{1 \mathrm{w}}\left(z_{\mathrm{s}}\right) .
\end{aligned}
$$

Regarding to the second harmonic component, Eq. (4) of Ref. 5) and Eq. (2) lead to

$$
P_{2 \mathrm{~W}}\left(z_{\mathrm{w}}\right)=\frac{\exp \left[j 2 g\left(z_{\mathrm{w}}\right) a^{2}\right]-1}{j 4 g\left(z_{\mathrm{w}}\right)} p_{2 \mathrm{w}}\left(z_{\mathrm{w}}\right) .
$$

Further one derives the following equation from Eq. (4) of Ref. 5):

$$
p_{2 \mathrm{w}}\left(z_{\mathrm{w}}\right)=\frac{\eta}{\zeta} \exp \left[-4 \alpha_{\mathrm{w}}\left(z_{\mathrm{w}}-z_{\mathrm{s}}\right)\right] p_{2 \mathrm{w}}\left(z_{\mathrm{s}}\right) .
$$

Then one obtains

$$
\begin{aligned}
P_{2 W}\left(z_{\mathrm{w}}\right)= & \frac{\exp \left[j 2 g\left(z_{\mathrm{w}}\right) a^{2}\right]-1}{j 4 \zeta g\left(z_{\mathrm{w}}\right)} \eta \\
& \cdot \exp \left[-4 \alpha_{\mathrm{w}}\left(z_{\mathrm{w}}-z_{\mathrm{s}}\right)\right] p_{2 \mathrm{w}}\left(z_{\mathrm{s}}\right),
\end{aligned}
$$

where

$$
\eta=\frac{\int_{0}^{z_{\mathrm{w}}} \frac{\exp \left(2 \alpha_{\mathrm{w}} z^{\prime}\right)}{h\left(z^{\prime}\right)} d z^{\prime}}{\int_{0}^{z_{\mathrm{S}}} \frac{\exp \left(2 \alpha_{\mathrm{w}} z^{\prime}\right)}{h\left(z^{\prime}\right)} d z^{\prime}} .
$$

Consequently $P_{2 \mathrm{w}}\left(z_{\mathrm{w}}\right)$ can be transformed to the axial pressure $p_{2 \mathrm{w}}\left(z_{\mathrm{s}}\right)$. On the other hand, in Ref. 5), the comparison between Eqs. (11) and (13) and that between Eqs. (12) and (14) give the following equations when $z=z_{\mathrm{s}}$. The above equation numbers are all those in Ref. 5).

$$
\begin{aligned}
& P_{1 \mathrm{~S}}\left(z_{\mathrm{s}}\right)=\frac{\exp \left[j g\left(z_{\mathrm{w}}\right) a^{2}\right]-1}{j 2 g\left(z_{\mathrm{w}}\right)} p_{1 \mathrm{~s}}\left(z_{\mathrm{s}}\right), \\
& P_{2 \mathrm{~S}}\left(z_{\mathrm{s}}\right)=\frac{\exp \left[j 2 g\left(z_{\mathrm{w}}\right) a^{2}\right]-1}{j 4 g\left(z_{\mathrm{w}}\right)} p_{2 \mathrm{~s}}\left(z_{\mathrm{s}}\right) .
\end{aligned}
$$

According to Eqs. (13) and (14), the concave receiver output at $z=z_{\mathrm{s}}$ for the case with an inserted sample is transformed to the axial pressure at the identical range $z=z_{\mathrm{s}}$.

Equations (8) and (11) give the phase parameter of the concave receiver output in the case without the insertion of the sample, $\Phi_{\mathrm{w}}\left(z_{\mathrm{w}}\right)=\angle P_{2 \mathrm{w}}\left(z_{\mathrm{w}}\right)-$ $2 \angle P_{1 \mathrm{w}}\left(z_{\mathrm{w}}\right)+\pi / 2$, as follows:

$$
\begin{aligned}
\Phi_{\mathrm{W}}\left(z_{\mathrm{w}}\right)= & \phi_{\mathrm{w}}\left(z_{\mathrm{s}}\right)+\angle\left[\exp \left\{j 2 g\left(z_{\mathrm{w}}\right) a^{2}\right\}-1\right] \\
& -2 \angle\left[\exp \left\{j g\left(z_{\mathrm{w}}\right) a^{2}\right\}-1\right] \\
& +\angle g\left(z_{\mathrm{w}}\right)+\frac{\pi}{2}+\angle \eta+\angle \zeta,
\end{aligned}
$$


where $\phi_{\mathrm{w}}\left(z_{\mathrm{s}}\right)$ is the axial phase parameter for the case without samples, which is expressed as $\phi_{\mathrm{w}}\left(z_{\mathrm{s}}\right)=\angle p_{2 \mathrm{w}}\left(z_{\mathrm{s}}\right)-2 \angle p_{1 \mathrm{w}}\left(z_{\mathrm{s}}\right)+\pi / 2$. The phase parameter for the case with a sample is derived similarly from Eqs. (13) and (14) as

$$
\begin{aligned}
\Phi_{\mathrm{S}}\left(z_{\mathrm{s}}\right)= & \phi_{\mathrm{s}}\left(z_{\mathrm{s}}\right)+\angle\left[\exp \left\{j 2 g\left(z_{\mathrm{w}}\right) a^{2}\right\}-1\right] \\
& -2 \angle\left[\exp \left\{j g\left(z_{\mathrm{w}}\right) a^{2}\right\}-1\right]+\angle g\left(z_{\mathrm{w}}\right)+\frac{\pi}{2},
\end{aligned}
$$

where $\phi_{\mathrm{s}}\left(z_{\mathrm{s}}\right)$ is the axial phase parameter expressed as $\phi_{\mathrm{s}}\left(z_{\mathrm{s}}\right)=\angle p_{2 \mathrm{~s}}\left(z_{\mathrm{s}}\right)-2 \angle p_{1 \mathrm{~s}}\left(z_{\mathrm{s}}\right)+\pi / 2$. After eliminating $g\left(z_{\mathrm{w}}\right)$ from Eqs. (15) and (16), one derives the following equation when using a relation of $\angle \eta+$ $\angle \zeta=\phi_{\mathrm{w}}\left(z_{\mathrm{w}}\right)-\phi_{\mathrm{w}}\left(z_{\mathrm{s}}\right)$.

$$
\phi_{\mathrm{s}}\left(z_{\mathrm{s}}\right)=\Phi_{\mathrm{s}}\left(z_{\mathrm{s}}\right)-\Phi_{\mathrm{w}}\left(z_{\mathrm{w}}\right)+\phi_{\mathrm{w}}\left(z_{\mathrm{w}}\right) .
$$

When the value of $\phi_{\mathrm{w}}\left(z_{\mathrm{w}}\right)$ is theoretically calculated, the experimental value for the axial phase parameter $\phi_{\mathrm{s}}\left(z_{\mathrm{s}}\right)$ is obtained from the measured values for $\Phi_{\mathrm{s}}\left(z_{\mathrm{s}}\right)$ and $\Phi_{\mathrm{w}}\left(z_{\mathrm{w}}\right)$. Then, since $\phi_{\mathrm{s}}\left(z_{\mathrm{s}}\right)$ is determined with the difference of two measured values, $\Phi_{\mathrm{s}}\left(z_{\mathrm{s}}\right)-\Phi_{\mathrm{w}}\left(z_{\mathrm{w}}\right)$, the same measurement error in $\Phi_{\mathrm{S}}\left(z_{\mathrm{s}}\right)$ and $\Phi_{\mathrm{w}}\left(z_{\mathrm{w}}\right)$, due to the frequency characteristic of the receiving sensitivity, never give rise to the error of $\phi_{\mathrm{s}}\left(z_{\mathrm{s}}\right)$.

\subsection{Evaluation of $R_{\mathrm{F}}$}

Using Eqs. (8), (9), (13), (14) and (17) in Sec. 2.1, the value of the measurement parameter $R_{\mathrm{F}}$ defined in Ref. 1) is obtained at $z=z_{\mathrm{s}}$ from the measured values of $\left|P_{1 \mathrm{w}}\left(z_{\mathrm{w}}\right)\right|, \quad\left|P_{2 \mathrm{w}}\left(z_{\mathrm{w}}\right)\right|, \quad \Phi_{\mathrm{w}}\left(z_{\mathrm{w}}\right), \quad\left|P_{1 \mathrm{~S}}\left(z_{\mathrm{s}}\right)\right|$, $\left|P_{2 \mathrm{~S}}\left(z_{\mathrm{B}}\right)\right|$ and $\Phi_{\mathrm{S}}\left(z_{\mathrm{s}}\right)$ at a certain source pressure amplitude $p_{0}$.

$$
\begin{aligned}
R_{\mathrm{F}}\left(z_{\mathrm{s}}\right)= & |\eta||\zeta|^{\alpha_{2} / 2 \alpha_{1}}\left|\frac{\boldsymbol{P}_{1 \mathrm{~W}}\left(z_{\mathrm{w}}\right)}{\boldsymbol{P}_{1 \mathrm{~S}}\left(z_{\mathrm{s}}\right)}\right|^{\left(\alpha_{2} / 2 \alpha_{1}\right)+1}\left|\frac{\boldsymbol{P}_{2 \mathrm{~S}}\left(z_{\mathrm{s}}\right)}{\boldsymbol{P}_{2 \mathrm{~W}}\left(z_{\mathrm{w}}\right)}\right| \\
& \cdot \frac{\sin \left[\Phi_{\mathrm{s}}\left(z_{\mathrm{s}}\right)-\Phi_{\mathrm{w}}\left(z_{\mathrm{w}}\right)+\phi_{\mathrm{w}}\left(z_{\mathrm{w}}\right)\right]}{\sin \phi_{\mathrm{w}}\left(z_{\mathrm{w}}\right)}
\end{aligned}
$$

where it is assumed that $\alpha_{\mathrm{w}}\left(z_{\mathrm{w}}-z_{\mathrm{s}}\right)=0$. The parameters $|\zeta|,|\eta|, \phi_{\mathrm{w}}\left(z_{\mathrm{w}}\right)$ and $\phi_{\mathrm{w}}\left(z_{\mathrm{s}}\right)$ are evaluated from Eqs. (7), (12) and the following two equations, respectively.

$$
\begin{aligned}
& \phi_{\mathrm{w}}\left(z_{\mathrm{w}}\right)=-\left[h\left(z_{\mathrm{w}}\right) \int_{0}^{z_{\mathrm{w}}} \frac{\exp \left(2 \alpha_{\mathrm{w}} z^{\prime}\right)}{h\left(z^{\prime}\right)} d z^{\prime}\right], \\
& \phi_{\mathrm{w}}\left(z_{\mathrm{s}}\right)=-\left[h\left(z_{\mathrm{s}}\right) \int_{0}^{z_{\mathrm{s}}} \frac{\exp \left(2 \alpha_{\mathrm{w}} z^{\prime}\right)}{h\left(z^{\prime}\right)} d z^{\prime}\right] .
\end{aligned}
$$

The examples of calculated $|\zeta|$ and $|\eta|$ are shown in Fig. 2. Supposing various sound speeds in the sample, the source dimension, frequency and sample

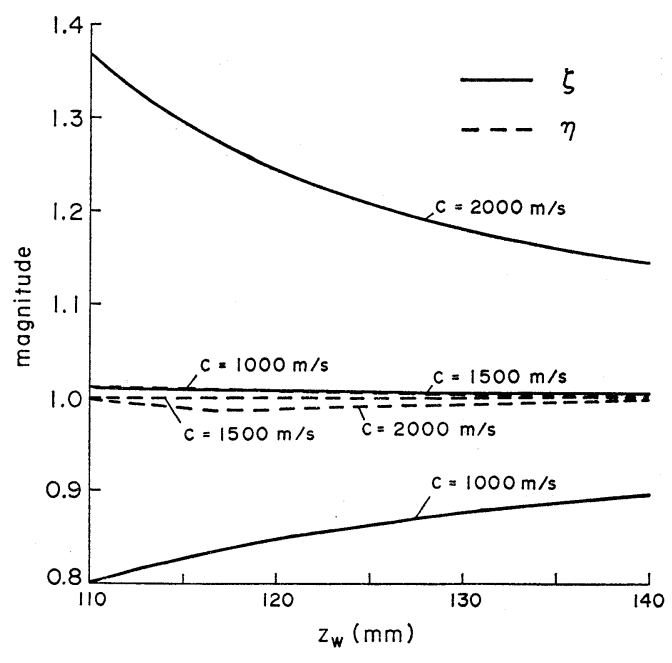

Fig. 2 Values of $|\eta|$ and $|\zeta|$ calculated for various maximum amplitude positions $z_{\mathrm{W}}$, which incorporate curvature radius of concave receiver.

thickness were assumed to be those in the experimental setup of $D=85 \mathrm{~mm}, \xi=2300 \mathrm{~m}^{-2}, f=1.9$ $\mathrm{MHz}$ and $L=20 \mathrm{~mm}$. The abscissa indicates the range for the maximum-amplitude $z_{\mathrm{w}} \approx D+d$ altered according to the curvature radius of concave receiver. Since $d=42 \mathrm{~mm}$ in the experiment described in the next section, it holds that $z_{\mathrm{w}} \approx 127 \mathrm{~mm}$. According to Fig. 2, since $|\eta|$ is always unity with an error below $1 \%,|\eta|$ is less effective to the value of $R_{\mathrm{F}}$.

The evaluation of $R_{\mathrm{F}}$ enables the measured data to be compared, as in Ref. 1), with the theoretical value of $R_{\mathrm{F}}$ assuming various $B / A$ values. The value of $B / A$ to make the value of $R_{\mathrm{F}}$ coincide with the measured $R_{\mathrm{F}}$ is found out to determine the value of the $B / A$.

\section{EXPERIMENT OF $B / A$ MEASUREMENT}

The experimental setup for testing the present $B / A$ measurement method is shown in Fig. 3. The sound source is a concave piezoelectric transducer with thickness resonant frequency of $1.9 \mathrm{MHz}$ and aperture radius of $40 \mathrm{~mm}$, whose rear electrode has been fabricated in star shape to approximate the Gaussian source of $\xi=2300 \mathrm{~m}^{-2}$. The focal length $D$ is $85 \mathrm{~mm}$. Another piezoelectric concave transducer with the thickness dilatational-mode resonance 


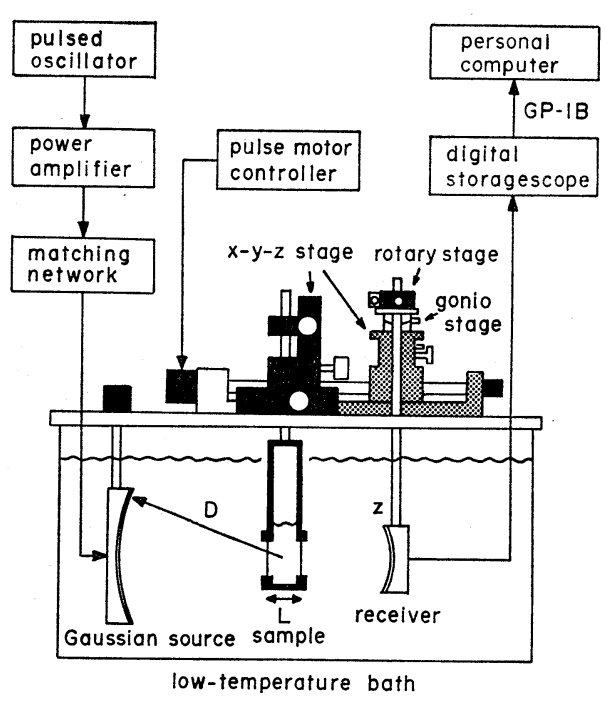

Fig. 3 Experimental setup.

frequency of $4 \mathrm{MHz}$, whose curvature and aperture radii are $d=42 \mathrm{~mm}$ and $a=20 \mathrm{~mm}$, respectively, is suspended with a brass rod attached to the mechanical stage, and is set against to the source. The stage is the combination of a rotary stage, a gonio stage, and an $x-y-z$ stage which facilitates the positioning of the $4-\mathrm{MHz}$ transducer to be used for the concave receiver. The sound source emits 1.9$\mathrm{MHz}$ burst waves with $21-\mu$ s duration time and $500-$ $\mathrm{Hz}$ repetition rate, in a low-temperature thermostatic water bath which keeps the temperature constant at $20^{\circ} \mathrm{C}$. The output voltage of the concave receiver is observed on a digital oscilloscope, and the waveform data are sent to a personal computer which executes an FFT to analyze the amplitudes and phases of first five harmonics. Only the results for the fundamental and second harmonic components are used in this experiment. A liquid sample inserted in the focal region is contained in a cubic case made from brass, which is attached to another $\mathrm{x}-\mathrm{y}-\mathrm{z}$ stage with a brass rod. The circular holes with the diameter of $17 \mathrm{~mm}$ on both sides of the case, normal to the $z$ axis, are the acoustic windows. The interval between the two windows determines the thickness of sample $L$ to be $20 \mathrm{~mm}$. To isolate the sample from water, polyvinyliden chloride films of $10-\mu \mathrm{m}$ thickness are used as the window materials.

Before the experiment, at the outside of water bath, the sample is set carefully such that the windows are parallel to the imaginary plane involving the edge of the concave transducer for the source and further the center of the windows locates at the symmetrical axis of the source. Then, in the water bath, while sliding the sample along the $z$ axis, the sample position to maximize the amplitude of a signal reflected at the water-sample interface observed with the source transducer itself is searched out. Since the incidence surface of the sample coincides with $z=D$ at this maximum position, the sample is then shifted toward the source by $L / 2=10$ $\mathrm{mm}$ so that the center of the sample locates at $z=D$.

Figures 4(a) and 4(b) compare the experimental and theoretical results for the $z$ dependence of the amplitudes and phase parameter in the case with the insertion of a methanol layer. The theoretical one has been calculated using the values of $\rho=795$ $\mathrm{kg} / \mathrm{m}^{3}$ and $c=1113 \mathrm{~m} / \mathrm{s}$ as measured below. Since the influence of the attenuation in methanol is small, it has been assumed that $\alpha_{1}=\alpha_{2}=0$. Further, so far as this calculation is concerned, the literature value 9.6 for $B / A$ has been employed. ${ }^{6)}$ The

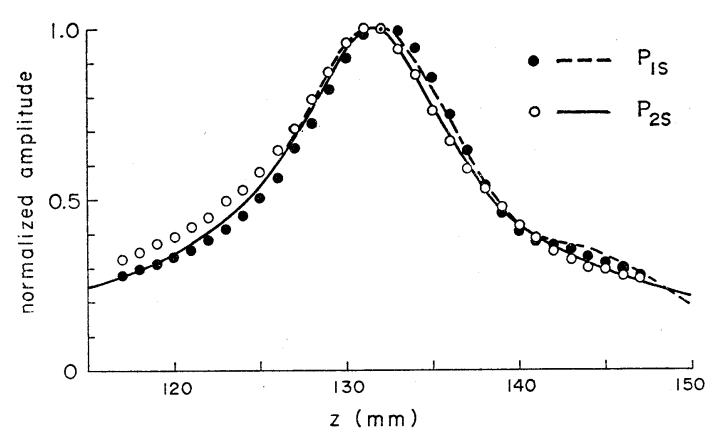

(a)

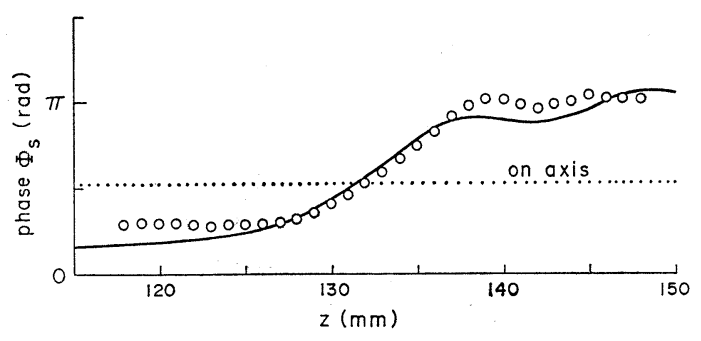

(b)

Fig. 4 Range dependence of received sound in the field with methanol layer. (a) Harmonic amplitudes. (b) Phase parameter. 
acoustic property of water has been assumed as $\rho_{\mathrm{w}}=1000 \mathrm{~kg} / \mathrm{m}^{3}, c_{\mathrm{w}}=1483 \mathrm{~m} / \mathrm{s}, \alpha_{\mathrm{w}}=0.1 \mathrm{~Np} / \mathrm{m}$ and $(B / A)_{\mathrm{w}}=5.0$. The experimental results show reasonable agreement with the theoretical ones. The range for the maximum amplitude becomes $5 \mathrm{~mm}$ far from the source compared to the case without any samples.5) This can be explained by $z_{\mathrm{s}}-z_{\mathrm{w}}=$ $-\left(c / c_{\mathrm{w}}-1\right) L$. At this maximum position $z_{\mathrm{g}}$, the phase parameter of the concave receiver output shown by a solid curve agrees with that of the axial pressure shown by a dotted curve.

The measured values for the amplitudes and phase parameter at the maximum-amplitude position are shown in Figs. 5(a) and 5(b) both in the case with-

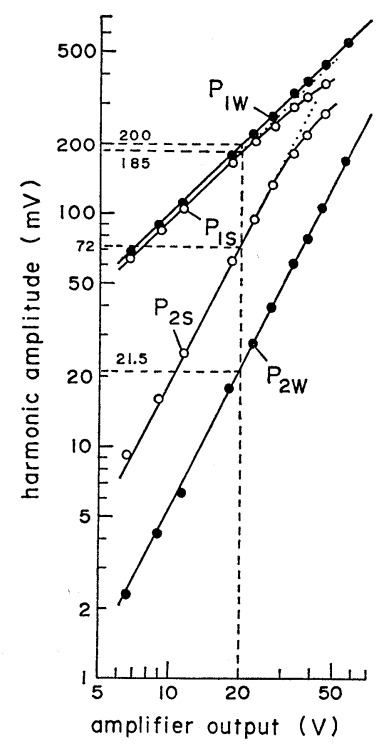

(a)

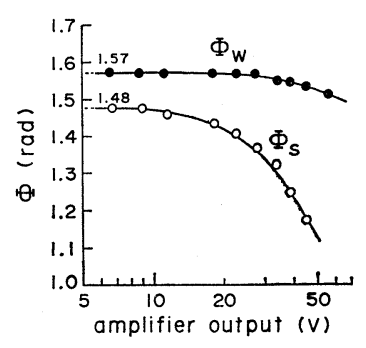

(b)

Fig. 5 Data obtained both for the cases with and without insertion of methanol sample. (a) Harmonic amplitudes. (b) Phase parameter. out and with the insertion of methanol layer. To evaluate the $R_{\mathrm{F}}$, the source pressure amplitude $p_{0}$ must be identical for two cases with and without insertion of a sample. The output voltage of an rf power amplifer to drive the transducer, $V_{0}$, was measured instead of $p_{0}$, for simplicity, and was taken as a parameter on the abscissa of Figs. 5(a) and 5(b).

The $R_{\mathrm{F}}$ is evaluated from Fig. 5 as follows. Adopting the measured amplitudes, for example, at $V_{0}=20 \mathrm{~V}, \quad\left|P_{1 \mathrm{~W}} / P_{1 \mathrm{~s}}\right|=200 / 185=1.081$ and $\left|P_{2 \mathrm{~S}}\right|$ $P_{2 \mathrm{~W}} \mid=72 / 21.5=3.35$. Further, adopting the phase parameter measured at small amplitudes, $\Phi_{\mathrm{w}}\left(z_{\mathrm{w}}\right)=$ 1.57 and $\Phi_{\mathrm{s}}\left(z_{\mathrm{s}}\right)=1.48$. From Eqs. (7), (12), (19) and (20), it is derived that $|\eta|=1.004,|\zeta|=0.8968$, $\phi_{\mathrm{w}}\left(z_{\mathrm{w}}\right)=1.69$ and $\phi_{\mathrm{w}}\left(z_{\mathrm{s}}\right)=1.67$ for the case that $z_{\mathrm{w}}=127 \mathrm{~mm}$ and $z_{\mathrm{s}}=132 \mathrm{~mm}$. Hence $\phi_{\mathrm{s}}\left(z_{\mathrm{s}}\right)=$ $1.48-1.57+1.69=1.60$ according to Eq. (17). Applying these values to Eq. (18), one obtains $R_{\mathrm{F}}\left(z_{\mathrm{s}}\right)=$ $1.004 \times 0.8968^{2} \times 1.081^{3} \times 3.35 \times \sin (1.60) / \sin (1.67)$ $=3.43$.

Figure 6 shows the relation between $R_{\mathrm{F}}$ at $z=132$ $\mathrm{mm}$ and $B / A$ of methanol sample whose $\rho, c$ and $\alpha_{1}$ are measured as below. The $\rho$ was measured to be $795 \mathrm{~kg} / \mathrm{m}^{3}$ using pycnometer, and $c$ was measured to be $1113 \mathrm{~m} / \mathrm{s}$ by the sing-around method employing the present experimental source and receiver. Since they are close to the literature values $\rho=791 \mathrm{~kg} / \mathrm{m}^{3}$ and $c=1121 \mathrm{~m} / \mathrm{s},{ }^{8)}$ it seems that the measurement is reasonable. On the other hand, the ratio of Eqs. (1) and (3) gives

$$
\frac{P_{1 \mathrm{~s}}}{P_{1 \mathrm{~W}}}=T_{\mathrm{I} 1} T_{\mathrm{O} 1} \exp \left[-\alpha_{1} L-\alpha_{\mathrm{w}}\left(z_{\mathrm{s}}-z_{\mathrm{w}}-L\right)\right] .
$$

Accordingly the measurement of the reduction of the

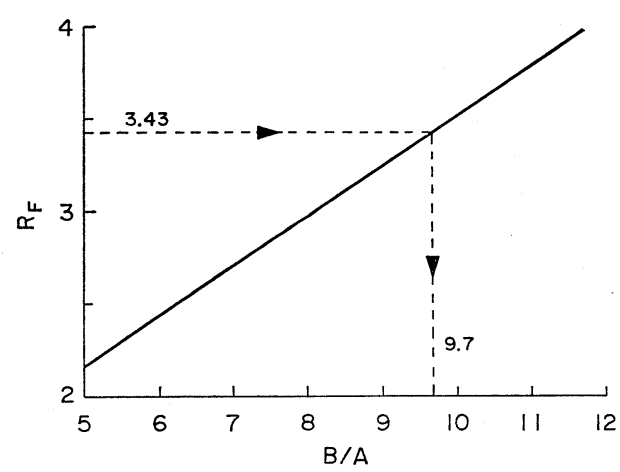

Fig. 6 Relation of $R_{\mathrm{F}}$ and $B / A$ at $z=132$ $\mathrm{mm}$, and determination of nonlinearity parameter $B / A$ of methanol. 


\section{S. SAITO: $B / A$ MEASUREMENT USING FOCUSED ULTRASOUND}

Table 1 Comparison of measured and literature values of $B / A$.

\begin{tabular}{lcc}
\hline \multicolumn{1}{c}{ Sample } & This study & Literature \\
\hline Methanol & 9.7 & 9.6 (Ref. 6) \\
& & 10.0 (Ref. 1) \\
Benzyl alcohol & 10.3 & 10.2 (Ref. 6) \\
& & 10.8 (Ref. 1) \\
$n$ Butyl alcohol & 11.1 & 10.7 (Ref. 6) \\
& & 11.2 (Ref. 1) \\
Glycerin & 9.6 & 8.8 (Ref. 9) \\
& & 9.3 (Ref. 10) \\
& & 9.4 (Ref. 1) \\
\hline
\end{tabular}

fundamental amplitude $\left|P_{1 \mathrm{~s}} / P_{1 \mathrm{w}}\right|$ due to the insertion of sample enables one to estimate $\alpha_{1}$ when the values of $T_{\mathrm{I} 1}, T_{\mathrm{O} 1}, z_{\mathrm{s}}-z_{\mathrm{w}}, \alpha_{\mathrm{w}}$ and $L$ are known. Assuming $\alpha_{1}=0$ and $\alpha_{\mathrm{w}}=0, T_{\mathrm{I} 1} T_{\mathrm{O}_{1}}$ is roughly evaluated as 0.936 . Substituting the experimental result $\left|P_{1 \mathrm{~S}} / P_{1 \mathrm{w}}\right|=0.925$ into Eq. (21), one obtains $\alpha_{1}=0.64 \mathrm{~Np} / \mathrm{m}$. The calculation for the relation of Fig. 6 was conducted with Eq. (19) of Ref. 1) using these values for $\rho, c$ and $\alpha_{1}$, where the relation $\alpha_{2}=4 \alpha_{1}$ was additionally employed. On the figure, it is seen that the $B / A$ to give $R_{\mathrm{F}}=3.43$ obtained experimentally is 9.7. This is the final measurement result for $B / A$. This value is comparable with $B / A=9.6$ in Ref. 6), and $B / A=10.0$ measured in Ref. 1) utilizing the axial pressure observation of focused sound. The measured value for $\alpha_{1}$ in methanol was larger than the literature value 0.12 $\mathrm{Np} / \mathrm{m} .{ }^{7)}$ The literature value may be correct. Nevertheless, since the $R_{F}$ value using the literature value for $\alpha_{1}$ decreases only by $0.13 \%$ at most compared to the values shown in Fig. 6 within the range of $5<B \mid A<12$, such a measurement error is not significant for the $B / A$ measurement.

Table 1 shows the comparison of the measured $B / A$ for liquid samples with the literature values, containing the result aforementioned. The agreement is fairly good in the case of alcohol samples. The measured $B / A$ for glycerin sample, whose attenuation is much larger than those in alcohol samples, is slightly larger than the $B / A$ in literatures. Although the precision is still questionable for such a dissipative sample, the possibility to measure the $B / A$ value is thoroughly demonstrated.

\section{CONCLUSION}

A method to measure the acoustic nonlinearity parameter $B / A$ of a sample inserted in the focal region, observing the focused ultrasound detected by a concave receiver, was presented. The validity of the present method was experimentally clarified.

In the present method, the amplitudes of the fundamental and second harmonic components and their phase parameter are measured at the receiver position, where the fundamental amplitude attains the maximum, both in the case without and with the insertion of the sample. All these measured values are transformed to the parameter values expected to appear in the axial pressure at the receiver position where the maximum amplitude is obtained in the case with the inserted sample. Then the previously presented parameter $R_{\mathrm{F}}$ defined by the axial pressures can be experimentally evaluated. On the other hand, the theoretical value for $R_{\mathrm{F}}$ is calculated by the manner that has been previously presented. The finding out the $B / A$ value, which makes the theoretical $R_{\mathrm{F}}$ value coincide with the experimental $R_{\mathrm{F}}$ value, leads to the determination of the $B / A$. The linear acoustic property of the sample necessary for the calculation of $R_{\mathrm{F}}$ is separately measured using the same experimental setup.

One of the purposes of the present study is to develop a technique to measure the nonlinearity parameter of the medium using an acoustic microscope. A microscope usually does not use a Gaussian source, but employs a source whose vibrational distribution is rather uniform. In addition, since ultrasounds with wavelength as short as $10 \mu \mathrm{m}$ are usually employed in acoustic microscopes, other problems associated with the precision of sample thickness and the roughness of sample surface may be outstanding in the $B / A$ measurement. The influence of sound attenuation enhanced at high frequency might also cause some additional difficulty, even if the source and sample could be shrinked in proportion to the wavelength. The present method, therefore, cannot be directly applied to the acoustic microscope. However, since the capability of concave receiver to be used for the $B / A$ measurement was clarified, the present method can be one step forward to the $B / A$ measurement with the acoustic microscope.

\section{ACKNOWLEDGMENTS}

The author wishes to thank the members of the Nonlinear Acoustics Research Society for invaluable 
discussion. Messrs. Yoshiyuki Okada, Kazuyoshi Ono, and Manabu Kitamura are thanked for their assistance in the experiment.

\section{REFERENCES}

1) S. Saito, "Measurement of the acoustic nonlinearity parameter in liquid media using focused ultrasound," J. Acoust. Soc. Am. 93, 162-172 (1993).

2) S. Saito, "A small-volume finite-amplitude method for nonlinearity parameter measurement using a focused Gaussian beam," Proc. 14th ICA, Beijin, C11-1 (1992).

3) S. Saito and B. C. Kim, "Selective detection of second harmonic sound generated at the focal region in a finite amplitude focusing field," $\mathbf{J}$. Acoust. Soc. Jpn. (E) 8, 165-175 (1987).

4) B. C. Kim and S. Saito, "Influence of inserted sample on second harmonic component in a finiteamplitude focused sound," J. Acoust. Soc. Jpn. (E) 10, 143-151 (1989).
5) S. Saito, "Second harmonic component of focused sound observed with a concave receiver," J. Acoust. Soc. Jpn. (E) 16, 29-36 (1995).

6) R. T. Beyer, Nonlinear Acoustics (U.S. Naval Sea Systems Command, Washington, D.C., 1974), p. 101.

7) O. Nomoto, "Sound absorption in liquids," in Cho-Onpa Gijutsu Binran (Handbook of Ultrasonic Technology), J. Saneyoshi, Y. Kikuchi, and O. Nomoto, Eds. (Nikkan-Kogyo-Shinbunsha, Tokyo, 1968), p. 1285 (in Japanese).

8) O. Nomoto, "Sound speed in liquids," ibid., p. 1211 (in Japanese).

9) L. K. Zarembo and V. A. Krasil'nikov, "Some problems in the propagation of ultrasonic waves of finite amplitude in liquids," Sov. Phys. Usp. 2, 580-599 (1959).

10) W. K. Law, L. A. Frizzell, and F. Dunn, "Ultrasonic determination of the nonlinearity parameter $B / A$ for biological media," Ultrasound Med. Biol. 11, 307-318 (1985). 